\title{
Robust nanopatterning by laser-induced dewetting of metal nanofilms
}

\author{
${ }^{1,2}$ Christopher Favazza, ${ }^{1,2}$ Ramki Kalyanaraman*, ${ }^{2,3}$ Radhakrishna Sureshkumar ${ }^{\dagger}$ \\ ${ }^{1}$ Department of Physics, Washington University in St. Louis, MO 63130 \\ ${ }^{2}$ Center for Materials Innovation, Washington University in St. Louis, MO 63130 \\ ${ }^{3}$ Department of Chemical Engineering, Washington University in St. Louis, MO 63130
}

\begin{abstract}
We have observed nanopattern formation with robust and controllable spatial ordering by laser-induced dewetting in nanoscopic metal films. Pattern evolution in Co film of thickness $1 \leq h \leq 8 \mathrm{~nm}$ on $\mathrm{SiO}_{2}$ was achieved under multiple pulse irradiation using a 9 ns pulse laser. Dewetting leads to the formation of cellular patterns which evolve into polygons that eventually break up into nanoparticles with monomodal size distribution and short range ordering in nearest-neighbour spacing $R$. Spatial ordering was attributed to a hydrodynamic thin film instability and resulted in a predictable variation of $R$ and particle diameter $D$ with $h$. The length scales $R$ and $D$ were found to be independent of the laser energy. These results suggest that spatially ordered metal nanoparticles can be robustly assembled by laser-induced dewetting.
\end{abstract}

*Corresponding author, ramkik@wuphys.wustl.edu

†E-mail: suresh@che.wustl.edu 


\section{Introduction}

Ordered metal nanoparticle arrays have become an active area of research due to their potential applications in nonlinear optics and nanophotonics below the diffraction limit [1-3]. However, practical realization of such applications require that cost-effective and reliable processes be developed for the manufacturing of ordered metal nanoarrays. Physical phenomena that lead to the formation of patterns with characteristic length scales and predictable time scales could be exploited in designing such nanomanufacturing processes. For instance, pattern formation following ion irradiation has been known to modify surface topography [4-8]. Rippling of surfaces under ion irradiation appears to be primarily due to an instability resulting from the competition between ion erosion and smoothening due to surface diffusion with the length and time scales determined by ion flux, surface temperature and surface diffusion parameters [9-12]. Another route to creating nanopatterns could be through instabilities of a thin fluid film leading to spatial patterns with length and time scales that depend on the thermophysical material properties such as interfacial tension, contact angle with the substrate, fluid viscosity and, for ultrathin films, long range dispersion forces such as the van der Waal's interaction. An example especially pertinent to thin film pattern formation is the hydrodynamic dewetting instability such as spinodal dewetting which occurs when attractive intermolecular forces exceed the stabilizing effect of interfacial tension [13-16]. Under such conditions, spontaneous film thickness fluctuations could be amplified and this will result in the breakup of the film leading eventually to the formation of drops/particles with well defined spatial order [17]. Dewetting dynamics leading to particles have been studied in detail in polymer films that are in the liquid state close to room temperature [18-21]. However, ordered nanoparticle formation via dewetting in metal films has remained relatively unexplored, primarily due to the high temperatures required to melt the metal and observe dewetting within practical time scales. Earlier work has shown that ion-irradiation of ultrathin Pt films on $\mathrm{SiO}_{2}$ substrates results in dewetting patterns with spatial order $[22,23]$. More recently our group has shown that multiple $n s$ pulsed laser melting of nanoscopic Co films can lead to short- and long-range spatial order [24-26]. However, the the relationship of particle spacing $R$ and diameter $D$ with film thickness $h$ was not determined. Furthermore, it is not clear whether thin film hydrodynamic models could help predict such relationships at nanometer length scales. Another unanswered question is whether dewetting under multiple cycles of phase change, i.e. melting and resolidification, would lead to robust final states. In this article, we address the above questions quantitatively by analyzing the results of a series of systematic experiments and interpreting them with the aid of thin film hydrodynamic theories. Such comprehensive understanding could help in knowledge-based design of nanomanufacturing facilities for ordered metal nanoarrays.

Specifically, we investigate the evolution of dewetting patterns in nanoscopic Co metal films 
on $\mathrm{SiO}_{2}$ in vacuum under multiple pulse irradiation with a laser of pulse length $\tau_{p}=9$ ns and wavelength $266 \mathrm{~nm}$. Co nanoparticles are useful towards nanoscale magnetic applications and in catalysis. Dewetting was studied as a function of film thickness $h$, laser energy density $E$ and laser irradiation time expressed in terms of the number of pulses $n$. We discovered that pattern formation evolved from cellular structures composed of polygons at early stages, which coalesced and eventually changed into nanoparticles at later times. Every observed pattern was ordered with a characteristic length scale while the nanoparticles had a well-defined nearest-neighbour $(N N)$ spacing $R$ and a monomodal size distribution with an average diameter $D$. The observed variations of $R$ and $D$ as function of $h$ were in agreement with the linear theory of spinodal dewetting. We also found that $R$ was independent of $n$ and $E$, provided net $C o$ evaporation was small. Based on these observations we infer that once the pulsed laser melting initiated dewetting, subsequent pulses evolved this instability while maintaining its spatial characteristics, thereby leading to a predictable final state. This offered strong evidence that the dewetting dynamics is extremely robust. To our knowledge this is the first experimental evidence for: (i) formation of metal nanoparticles with spatial order via a hydrodynamic dewetting instability; (ii) evidence for the linear spinodal theory under multiple instances of phase change; and (iii) evidence for robustness of the dewetting process to experimental conditions.

\section{Experimental Details}

Co films with thickness $\sim 1$ to $8 \mathrm{~nm}$ were deposited at rates varying between 0.5 to $2 \mathrm{~nm} / \mathrm{min}$ onto commercial optical quality $\mathrm{SiO}_{2} / \mathrm{Si}$ wafers consisting of $400 \mathrm{~nm}$ thick thermally grown oxide layer on polished $S i(100)$ wafers under high vacuum $\left(2 \times 10^{-8} \mathrm{Torr}\right)$ by e-beam evaporation at room temperature $[24,26]$. For every film thickness we measured the surface roughness and average grain size. Atomic force microscopy (AFM) of the surface roughness established an upper limit of $0.1 \pm .03 \mathrm{~nm}$ average $\mathrm{rms}$ roughness for the entire thickness range while transmission electron microscopy gave a average grain size of $\sim 20 \mathrm{~nm}$. No systematic change in roughness or particle size was detected with increasing film thickness. Further, no spatially ordered defects or other heterogeneities were observed on the native substrate surface. For the film thickness range investigated the laser absorption at $266 \mathrm{~nm}$ wavelength was uniform within the Co film (absorption depth $\sim 11 \mathrm{~nm})$. Therefore vertical temperature gradients could be neglected and the entire film temperature may be modeled as a uniform, time varying function that depends on the laser power (source term) and conduction of heat to the substrate (sink term) [27]. The film thickness was measured by concentration measurements of Co via energy dispersive X-ray spectrometry (EDS) in a scanning electron microscope (SEM). The concentration was converted into an equivalent thickness value by using calibration based on step-height and Rutherford backscattering measure- 
ments of the film thickness. The films were irradiated immediately after deposition in vacuum at normal incidence by an unfocused laser beam of area $3 \times 3 \mathrm{~mm}^{2}$ at a repetition rate of $50 \mathrm{~Hz}$. Under these conditions, the film surface was never exposed to air. Hence no oxide layer was expected to form on the film surface. Pattern formation was studied for energy densities $E$ between $40 \leq E \leq 150 \mathrm{~mJ} / \mathrm{cm}^{2}$ and for $n$ between 10 and 10,500 pulses. For each thickness the range of $E$ was chosen to be above the melt threshold. This was determined by a visible roughening of the metal film surface, as detected under high-resolution SEM within the longest time scale of the experiment (i.e. after 10,500 laser pulses) [28]. Analytical estimates based on a 1-D heat transport model as well as finite element simulations showed that the lifetime $\tau_{m}$ of the Co liquid ranged from $\sim 2 \leq \tau_{m} \leq 12 n s$ in the entire $h$ range studied [27]. The same model predicted cooling rates of the order of $10^{10} \mathrm{~K} / \mathrm{s}$ and a total heating plus cooling time per pulse of $\sim 100 \mathrm{~ns}$, which was much smaller than the spacing between pulses of $20 \mathrm{~ms}$. Hence, during each pulse, the film melts and in the molten state, dewetting process occurs. Upon the cessation of the pulse, the film solidifies within a time scale much smaller than the interval between subsequent pulses. The maximum temperature of the molten liquid above the Co melting point $T_{m}=1768 \mathrm{~K}$ was estimated to be $\sim 500 K$. Under these conditions, the maximum evaporation observed after the longest $n$ at the highest $E$ was $\leq 15 \%$.

\section{Results}

Fig. 11 shows the dewetting morphology observed for irradiation at $E=120 \mathrm{~mJ} / \mathrm{cm}^{2}$ of a $3.8 \mathrm{~nm}$ thick Co film for increasing number of laser pulses $n$. This evolution sequence is typical of the ordered morphology observed in the thickness range of $1 \leq h \leq 8 \mathrm{~nm}$. After a few pulses, the dewetting morphology is characterized by a cellular network of polygons (Fig. 11A). As $n$ is increased the metal/substrate contact lines recede to the edge of the holes resulting in a network of coalescing polygonal features (Fig. 1B). Continued irradiation results in the formation of nanoparticles primarily at the junctions of the polygons, as evident from the location of the particles in Fig. $1 \mathrm{~B}$ and in $\mathrm{C}$ and D. At every observed stage, a characteristic length scale $L$ was present, as evidenced by the annular form of the power spectrum of the spatial correlations in the intensity variation within each pattern. The power spectrum is shown alongside the corresponding images in Fig. 174 to D. For patterns consisting of polygons, $L$ represents the average distance between the center to the vertices, while in the case of the nanoparticles it represents the average $N N$ spacing $R$.

A nonlinear peak fitting algorithm (Levenberg-Marquardt) was used to locate Gaussian shaped peaks in the radial distribution function $g(k)$ versus the wavenumber $k \equiv 1 / L$ for each pattern (Fig. 2A). The variation of $L$ with $n$ for various $E$ values is shown in Fig. $2 \mathrm{~B}$ (the lines are only 
guides for the eye). The initial rapid increase in $L$ corresponds to the increase in the average size of the polygons. The decrease corresponds to the breakup of polygons into nanoparticles, with the long time value representing $R$. The size distribution of nanoparticles in the final state was observed to be monomodal, as shown in Fig. $2 \mathrm{C}$. Also, as seen from Fig. $2 \mathrm{~B}$, the value of $R$ remains unchanged over long irradiation times and is independent of laser energy $E$. However, increasing $E$ increases the rate at which the patterns evolve, as evident from Fig. $2 \mathrm{~B}$ and the figure in the inset. At the lowest energy of $110 \mathrm{~mJ} / \mathrm{cm}^{2}$ the time to reach $R$ ( $n>3000$ ) was observed to be much larger than the $130 \mathrm{~mJ} / \mathrm{cm}^{2}$ case $(n \leq 3000)$. Since the temporal scale used here is coarse, i.e., it is expressed in number of pulses, the precise location of the peak value of $L$ was not detectable for every energy value.

\section{Discussion}

It is well accepted that dewetting morphology can progress via three pathways [29]: (i) Homogeneous nucleation and growth, where holes appear spontaneously at random locations and times on the surface. Because of the inherent randomness, no characteristic length is present in this type of dewetting [20]; (ii) Heterogeneous nucleation and growth due to defects, impurities or other experimentally imposed heterogeneities. In this type of dewetting, a characteristic length scale could appear at the early stages of dewetting due to ordered nucleation sites. For instance, in ionirradiation induced dewetting, the average molten zone of an ion imposes a characteristic length scale in dewetting [23]; and (iii) Thin film hydrodynamic (T.F.H.) instabilities such as the one associated with the dewetting of spinodally unstable systems. The resulting patterns are characterized by a well-defined length scale in the hole spacing and/or size [19]. Since we have ruled out spatially ordered heterogeneities on the surface and in the film microstructure, the evidence for spatial ordering in Fig. 1 strongly suggests spinodal dewetting.

We examined whether T.F.H. models developed based on lubrication analysis could help explain the time and length scales of the observed dewetting patterns. Towards this end, we adopted the T.F.H. equations employed by Vrij $[13,30]$ and Sharma and Ruckenstein [14] to study spinodal dewetting in ultrathin polymeric films. Lubrication theory provides a relationship between the mass flux (which can be related to temporal variations in film height) and the effective pressure gradient, which for a thin film could result from perturbations that produce interface curvature, and disjoining pressure due to long-range intermolecular forces such as van der Waals interactions. For a thin film resting on a substrate contribution to the disjoining pressure can be expressed as $A / 6 \pi h^{\prime 3}$ where $A$ is the Hamaker constant and $h^{\prime}$ is the instantaneous film thickness. This approach 
leads to the dynamical equation describing the film height as a function of time as:

$$
3 \eta \frac{\partial h^{\prime}}{\partial t}=-\nabla \cdot\left(\gamma h^{\prime 3} \nabla \cdot \nabla^{2} h^{\prime}+\frac{A *}{h^{\prime}} \nabla h^{\prime}\right)
$$

where $\eta$ is the viscosity and $\gamma$ is the interfacial energy of the film-vacuum interface and $A * \equiv$ $A / 2 \pi$. If infinitesimally small perturbations $\hat{h}$ are imposed on an initially flat film of uniform thickness $h$, i.e. $h^{\prime}=h+\hat{h}$, the growth/decay rate of such perturbations can be assessed by using linear stability analysis in which one keeps only terms which are linear in $\hat{h}$ :

$$
3 \eta \frac{\partial \hat{h}}{\partial t}=-\nabla \cdot\left(\gamma h^{3} \nabla \cdot \nabla^{2} \hat{h}+\frac{A *}{h} \nabla \hat{h}\right) .
$$

Substitution of the normal mode form $\hat{h}=\epsilon \exp \left(i k_{x} x+i k_{y} y+\sigma t\right)$, where $x$ and $y$ denote the orthogonal surface coordinates and $\sigma$ represents the growth or decay rate of perturbations with the characteristic wavevector $\mathbf{k} \equiv\left(k_{x}, k_{y}\right)$, into eq. 2 gives the following dispersion relationship $\left(k^{2} \equiv \mathbf{k} \cdot \mathbf{k}\right)$ :

$$
3 \eta \sigma=-\gamma h^{3} k^{4}+\frac{A *}{h} k^{2}
$$

It is evident from eq. 3 that $\sigma>0$ for perturbations with wavevectors with $k^{2}<\frac{A *}{\gamma h^{4}}$ implying that such perturbations will be amplified as time progresses. The fastest growing mode $k_{m}$ can be obtained by letting $\frac{\partial \sigma}{\partial k}=0$. This gives $k_{m}^{2}=\frac{A *}{2 \gamma h^{4}}$. Hence, the characteristic wavelength $\Lambda \equiv 2 \pi / k_{m}$ is given by:

$$
\Lambda=\sqrt{\frac{16 \pi^{3} \gamma}{A}} h^{2} .
$$

The time scale $\tau_{D} \equiv 2 \pi / \sigma$ associated with the growth of perturbations with wavelength $\Lambda$ can be evaluated by letting $k=k_{m}$ in eq. [3] as:

$$
\tau_{D}=\frac{96 \pi^{3} \gamma \eta h^{5}}{A^{2}}
$$

A previous investigation by Bischof et al. using single shot ns pulsed laser melting of a multilayer metal structure has shown that is possible to capture snapshots of a dewetting instability provided the characteristic time scale of the dewetting process is much larger than the liquid melt time $\tau_{m}[31,32]$. Based on our analytical model the liquid lifetimes were estimated to be $2 \leq \tau_{L} \leq 12$ ns for the range of experimental parameters reported here [27]. Further, using eq. 5] we estimated the dewetting time scale at the melting point $T_{\text {melt }}$ of Co using $\gamma^{C o}\left(T_{m}\right)=1.88 \mathrm{~J} / \mathrm{m}^{2}$, viscosity $\eta^{C o}\left(T_{m}\right)=4.46 \times 10^{-3} \mathrm{~Pa}-s$, and a typical range of the Hamaker constant $A$ for metals from $10^{-20} \leq A \leq 10^{-18} \mathrm{~J}$. We found that the lower limit is $\tau_{D} \sim 25 \mathrm{~ns}$ for a $1 \mathrm{~nm}$ thick film and this time increases rapidly with increasing $h$. Therefore, it is reasonable to expect that pulsed 
laser melting used in this experiment could capture snapshots of spinodal dewetting. We further suggest that once the dewetting instability is initiated, continued laser pulsing fosters the instability eventually leading to a robust final state whose length scale, as suggested by eq. 4, should be independent of the laser parameters. This effect is observed in Fig. 2 $\mathrm{B}$, where despite a change in laser $E$ and/or $n$, an identical $N N$ spacing $R$ is observed in the stable nanoparticle state.

To provide further quantitative evidence for spinodal dewetting, we have investigated the experimental variation of the $N N$ particle spacing $R$ with $h$. The origin of the space-filling polygonal shapes can be explained by the presence of the preferred spinodal wavenumber $k \sim \frac{1}{\Lambda}$, which is a scalar measure of the wavevectors $k_{x}$ and $k_{y}$ in the two orthogonal directions along the undisturbed planar film, defined as $k=\sqrt{k_{x}^{2}+k_{y}^{2}}$. Since there are no external length scales in the $x$ and $y$ directions, one could have various combinations of $k_{x}$ and $k_{y}$ leading to the observed shapes [33]. Therefore the result of the linear spinodal theory (eq. 4) suggests that the polygon characteristics such as size, center-to-center spacings and the vertex separations will be proportional to $h^{2}$. As we have shown from Fig. 1 the observed nanoparticles form predominantly at the polygon vertices. Therefore it is clear that if the nanoparticle spacing is a result of spinodal dewetting, then $R$ should be proportional to $\Lambda$ and also vary as $h^{2}$. In Fig. 2(D), the experimentally observed variation of $R$ (solid circles) with $h$ is plotted. A power law fit to the variation in $R$ yields $R=25.7 h^{1.98 \pm 0.3}$ where the exponent of $h$ is in excellent agreement with the prediction of eq. 4 of 2 . The $N N$ spacing varied from $\sim 50$ to $1000 \mathrm{~nm}$ for the films studied. From the prefactor of $25.7 \mathrm{~nm}^{-1}$, the Hamaker constant was estimated as $A=14.1 \times 10^{-19} \mathrm{~J}$ (assuming the proportionality factor correlating $R$ to $\Lambda$ is 1 ). This value is of the right sign and order of magnitude for metals in general [34]. We also evaluated the average diameter $D$ of the nanoparticles as a function of $h$ (Fig. 2D open squares). $D$ varied from $\sim 30$ to $250 \mathrm{~nm}$ for the thickness range studied here. Using a volume conservation argument $D$ should vary with $h$ as $D \sim h^{5 / 3}$ [18]. The power-law fit to our experimental data (Fig. 2D) gave $D=14.1 h^{1.6 \pm 0.3}$ where the exponent is once again in good agreement with the theoretical prediction. These results clearly support a dewetting mechanism caused by a thin film hydrodynamic instability and also provides evidence that the linear spinodal theory is applicable under experimental conditions in which multiple cycles of film melting and solidification are present.

The break-up of the polygonal structures into droplets requires some elaboration. Previous studies of break-up of cellular structures into particles have attributed the process to a Rayleigh instability [35] as a result of which the particle diameter will follow the trend $D \sim h^{3 / 2}[18,36]$. However, our interpretation of the experimental observations reported here is that particle formation occurs via progression of the spinodal dewetting instability and through capillary effects. The primary evidence that supports this interpretation is that the nanoparticles predominantly form at the junctions of the polygons, as compared to the classical string-like particle distribution result- 
ing from the Rayleigh instability of the polygon sides [18]. Capillary effects resulting from the difference in curvature of the spheroidal particles at the junctions and the polygon walls will also act to drive the liquid towards the junction, a situation that is relatively well understood from the classical problem of dragging of a liquid film by a moving plate [37].

For the spinodal process it is possible to rationalize why $R$ is invariant to laser energy $E$ despite the increase in the rate of dewetting $\sigma \propto L / n$, as observed from Fig. 2(B) for early stages (small values of $n$ ). The increase in rate with increasing $E$ is due to two effects: one is the increase in liquid lifetime $\tau_{L}$ which will prolong the dewetting process within a given number of pulses; the second is the increase in liquid temperature $T_{L}$ above $T_{m}$ which will increase the rate as:

$$
\Delta \sigma(\Delta T)=-\sigma\left(T_{m}\right)\left[\frac{1}{\gamma\left(T_{m}\right)} \frac{d \gamma}{d T}+\frac{1}{\eta\left(T_{m}\right)} \frac{d \eta}{d T}\right] \Delta T
$$

where the Hamaker constant was treated as T-independent based on its extremely weak Tdependence [38]. Since the the T-dependent material parameters for Co of $d \gamma / d T=-0.5 \times$ $10^{-3} \mathrm{~J} / \mathrm{m}^{2}-s$; and $d \eta / d T=-6.3 \times 10^{-6} \mathrm{~Pa}-\mathrm{s} / \mathrm{K}$ both decrease with increasing $\mathrm{T}$, therefore, the rate increases with increasing $T$, as qualitatively observed from the inset of Fig. 2] B). This increase will be reinforced by that produced by the longer melt lifetime. A similar analysis shows that the dewetting length scales $L \propto \Lambda$ will decrease with increasing $\Delta T$ as:

$$
\Delta L(\Delta T)=\frac{L\left(T_{m}\right)}{2 \gamma\left(T_{m}\right)} \frac{d \gamma}{d T} \Delta T=-2.66 \times 10^{-4} L\left(T_{m}\right) \Delta T
$$

This decrease in $L$ with increasing $T_{L}$ can be estimated to be $\sim-13 \%$ for $\Delta T=500 \mathrm{~K}$, which is a relatively small change and will be undetectable given the larger spread in the experimental values of $R$ of $\pm 20 \%$ as measured from the $g(k)$. Therefore, for conditions under which the net Co evaporation is small, the changes in $R$ will be experimentally indistinguishable.

\section{Conclusion}

In conclusion, we studied pattern evolution in nanoscopic Co films $(1 \leq h \leq 8 \mathrm{~nm})$ under irradiation by multiple $9 \mathrm{~ns}$ laser pulses. Laser melting initiates a dewetting instability and subsequent irradiation fosters this dewetting to a stable state of spatially ordered nanoparticles. The dewetting instability appears to be spinodal in nature based on the observations of a characteristic length scale at all stages of dewetting and the $\sim h^{2}$ behavior of the $N N$ spacing of the nanoparticles. To our knowledge this is the first experimental evidence for: (i) formation of metal nanoparticles with spatial order via spinodal dewetting; (ii) evidence for linear spinodal theory under multiple cycles of phase change; and (iii) evidence for robustness of the dewetting process with respect to 
experimental conditions. The short range order and particle size produced by the pattern selection mechanisms can be manipulated by employing films with varying thickness. This offers opportunities to develop cost-effective and easily controllable nanomanufacturing processes for metal nanoarrays.

RK acknowledges support by the National Science Foundation through grant \# DMI-0449258. The authors acknowledge invaluable comments provided by Prof.'s Ken Kelton and Linday Greer during the preparation of this manuscript.

\section{References}

[1] M. Quinten, A. Leitner, J.R. Krenn, and F.R. Aussenegg. Electromagnetic energy transport via linear chains of silver nanoparticles. Optics Lett., 23(17):1331-33, 1998.

[2] B. Lamprecht, G. Schider, R. T. Lecchner, H. Ditlbacher, J. R. Krenn, A. Leitner, and F. R. Aussenegg. Metal Nanoparticle Gratings: Influence of Dipolar Particle Interaction on the Plasmon Resonance. Phys. Rev. Lett., 84:4721-4724, 2000.

[3] S.A. Maier, P.G. Kik, H.A. Atwater, S. Meltzer, E. Harel, B.E. Koel, and A.A.G. Requicha. Local detection of electromagnetic energy transport below the diffraction limit in metal nanoparticle plasmon waveguide. Nature Materials, 2:229, 2003.

[4] Jonah Erlebacher, Michael J. Aziz, Eric Chason, Michael B. Sinclair, and Jerrold A. Floro. Nonclassical smoothening of nanoscale surface corrugations. Physical Review Letters, 84(25):5800-5803, 2000.

[5] S. Facsko and H Kurz. Energy dependence of quantum dot formation by ion sputtering. Phys. Rev. B, 63:165329-1-165329-5, 2001.

[6] R. Moroni, D. Sekiba, F. Buatier de Mongeot, G. Gonella, C. Boragno, L. Mattera, and U. Valbusa. Uniaxial magnetic anisotropy in nanostructured co/cu(001): From surface ripples to nanowires. Physical Review Letters, 91(16):167207, 2003.

[7] M. Xu and C. Teichert. How do nanoislands induced by ion suputtering evolve during the early stage of growth? Journal of Applied Physics, 96(4):2244-2248, 2004.

[8] Alessandro Molle, F. Buatier de Mongeot, C. Boragno, R. Moroni, F. Granone, D. Sekiba, R. Buzio, U. Valbusa, R. Felici, and C. Quiros. Dense arrays of co nanocrystals epitaxially grown on ion-patterned cu(110) substrates. Applied Physics Letters, 86(14):141906, 2005.

[9] P. Sigmund. J. Mat. Sci., 8:1545, 1965. 
[10] R.M. Bradley and J.M.E. Harper. Theory of ripple topography induced by ion bombardment. J. Vac. Sci. Tech. A, 6:2390-95, 1988.

[11] E. Chason, T. M. Mayer, B. K. Kellerman, D. T. Mcllroy, and A. J. Howard. Roughening instability and evolution of the Ge(001) surface during ion sputtering. Phys. Rev. Lett., 72(19):3040-3043, 1994.

[12] Jonah Erlebacher, Michael J. Aziz, Eric Chason, Michael B. Sinclair, and Jerrold A. Floro. Spontaneous pattern formation in ion bombarded Si(001). Phys. Rev. Lett., 82(11):23302333, 1999.

[13] A. Vrij. Possible mechanism for the spontaneous rupture of thin, free liquid films. Discuss. Faraday Soc., 42:23-27, 1966.

[14] A. Sharma and E. Ruckenstein. Finite-Amplitude Instability of Thin Free and Wetting Films: Prediction of Lifetimes. Langmuir, 2:480-494, 1986.

[15] Ashutosh Sharma. Relationship of thin film stability and morphology to macroscopic parameters of wetting in the apolar and polar systems. Langmuir, 9(3):861-869, 1993.

[16] R. Seemann, S. Herminghaus, and K. Jacobs. Dewetting patterns and molecular forces. Phys. Rev. Lett., 86:5534-37, 2001.

[17] F. Brochard Wyart and J. Daillant. Drying of solids wetted by thin liquid films. Can. J. Phys., 68:1084-88, 1990.

[18] G. Reiter. Dewetting of thin polymer films. Phys. Rev. Lett., 68(1):75-78, 1992.

[19] Uwe Thiele, Michael Mertig, and Wolfgang Pompe. Dewetting of an evaporating thin liquid film: heterogeneous nucleation and surface instability. Phys. Rev. Lett., 80(13):2869-2872, 1998.

[20] T.G. Stange and D.F. Evans. Nucleation and growth of defects leading to dewetting of thin polymer films. Langmuir, 13:4459-4465, 1997.

[21] Uwe Thiele, Manuel G. Velarde, and Kai Neuffer. Dewetting: film rupture by nucleation in the spinodal regime. Phys. Rev. Lett., 87(1):016104, 2001.

[22] X. Hu, D.G. Cahill, and R.S. Averback. Nanoscale pattern formation in Pt thin films due to ion-beam-induced dewetting. Appl. Phys. Lett., 76:3215-17, 2000.

[23] X. Hu, D. G. Cahill, and R. S. Averback. Dewetting and nanopattern formation of thin Pt films on $\mathrm{SiO} 2$ induced by ion beam irradiation. J. Appl. Phys., 89:7777-83, 2001. 
[24] C. Favazza, J. Trice, H. Krishna, and R. Kalyanaraman. Laser-induced patterning of Co nanostructures under ambient conditions. In Mat. Res. Soc. Symp. Proc., volume 890, pages 0890-Y04-06.1-06.6, Pittsburgh, PA, 2006.

[25] C Favazza, J. Trice, A.K. Gangopadhyay, H. Garcia, R. Sureshkumar, and R. Kalyanaraman. Nanoparticle ordering by dewetting of Co on SiO2. J. Elec. Mat, August 2006.

[26] C. Favazza, J. Trice, H. Krishna, R. Kalyanaraman, and R. Sureshkumar. Laser-induced short- and long-range ordering of Co nanoparticles on SiO2. Appl. Phys. Lett., 88:153118183, 2006.

[27] J. Trice, D.G. Thomas, C. Favazza, R. Sureshkumar, and R. Kalyanaraman. Investigation of laser-induced dewetting in nanoscopic Co films: Experiments and modeling of thermal behavior. 2006.

[28] E. Matthias, M. Reichling, J. Siegel, O. W. Kading, S. Petzoldt, H. Skurk, P. Bizenberger, and E. Neske. The influence of thermal diffusion on laser ablation of metal films. Appl. Phys. A, 58:129-136, 1994.

[29] Pierre-Gilles de Gennes, Francoise Brochard-Wyart, and David Quere. Capillarity and Wetting Phenomenon. Springer, New york, 2003.

[30] A. Vrij and J. Th. G. Overbeek. Rupture of Thin Liquid Films Due to Spontaneous Fluctuations in Thickness. J. Am. Chem. Soc., 90:3074-78, 1968.

[31] J. Bischof, D. Scherer, S. Herminghaus, and P. Leiderer. Dewetting modes of thin metallic films: nucleation of holes and spinodal dewetting. Phys. Rev. Lett., 77(8):1536-1539, 1996.

[32] J. Bischof, M. Reinmuth, Johannes Boneberg, Stephan Herminghaus, T. Palberg, and Paul Leiderer. Behavior of thin metallic films melted with a nanosecond laser pulse. volume 2777, pages 119-127. SPIE, 1996.

[33] S. Chandrasekhar. Hydrodynamic and Hydromagnetic stability. Dover publications, NY, 1981.

[34] J.N. Israelachvili. Intermolecular and surface forces, chapter van der Waals forces between surfaces. Academic Press, London, UK.

[35] L. Rayleigh. On the Capillary Phenomena of Jets. Proc. London Math. Soc., 10:4, 1879.

[36] S. J. Henley, J. D. Carey, and S. R. P. Silva. Pulsed-laser-induced nanoscale island formation in thin metal-on-oxide films. Phys. Rev. B, 72:195408-I-195408-10, 2005. 
[37] L.D. Landau and B.G. Levich. Dragging of a liquid film by a moving plate. Acta Physicochim. U.R.S.S., 17:42-54, 1942.

[38] R.H. French. Origins and applications of London dispersion forces and Hamaker constants in ceramics. J. Amer. Ceram. Soc., 83:2117-46, 2000. 


\section{Figure captions}

- Figure 1. Scanning electron micrgraphs of dewetting patterns as a function of increasing number of laser pulses $n$ for a $3.8 \mathrm{~nm}$ thick $C o$ film at a laser energy density of $120 \mathrm{~mJ} / \mathrm{cm}^{2}$. (A) Polygons after $n=10$; (B) Coalescing polygons with evidence for particles at the junctions after $n=500$; (C) Nanoparticles with some remaining evidence of the polygon edges after $n=3000$; and (D) The final nanoparticle morphology after $n=10500$. The inset of each figure shows the corresponding power spectra for each pattern.

- Figure 2,Evidence for the characteristic length scales and their behavior for the dewetting process. (A) The $g(k)$ (symbols) and fits (lines) for the patterns of Fig. 1 (B) Dependence of $L$ on $n$ at various laser energies for the $3.8 \mathrm{~nm}$ film. The dashed lines are only intended to be guides for the eye. The inset shows the change in L for various energies at early stages, as measured after 10 pulses. (C) The monomodal size distribution of nanoparticles in the final state for the $3.8 \mathrm{~nm}$ film. (D) Nearest-neighbour spacing $R$ (solid circles) and Diameter $D$ (open squares) of the nanoparticles vs. film thickness $h$ and the least-squares fit. 


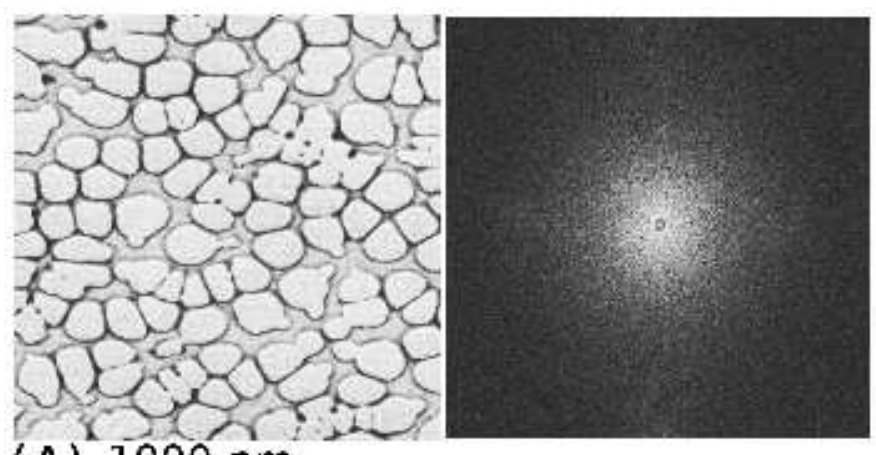

(A) $1000 \mathrm{~nm}$

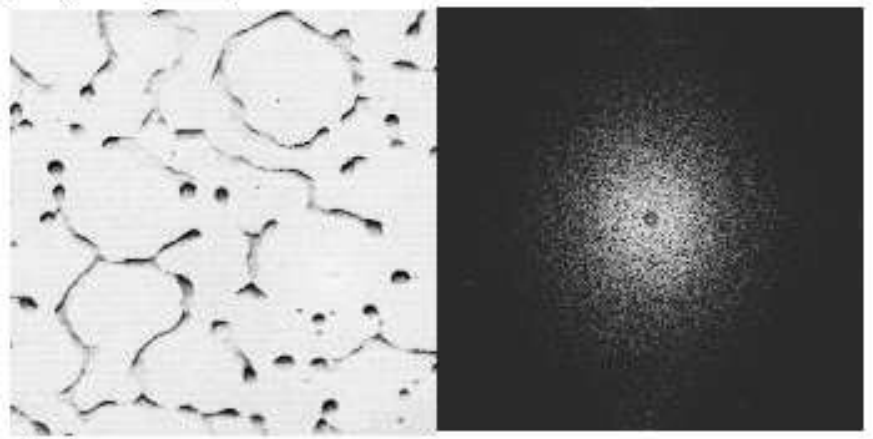

(B) $1000 \mathrm{~nm}$

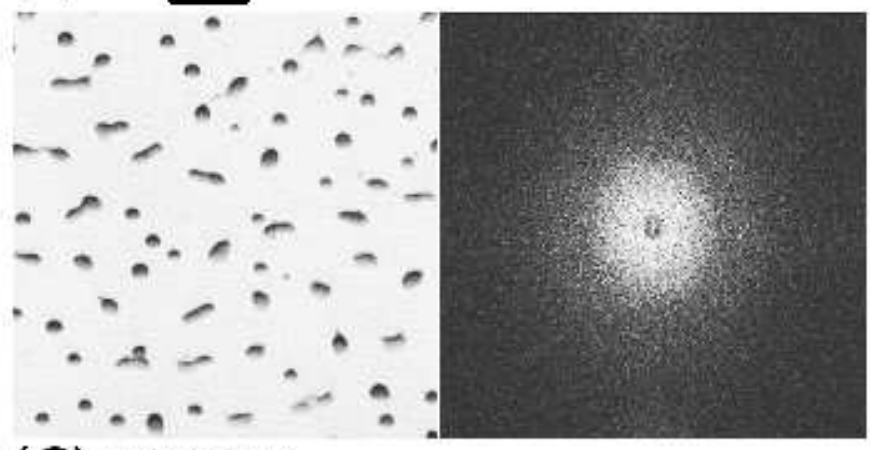

(C) $1000 \mathrm{~nm}$

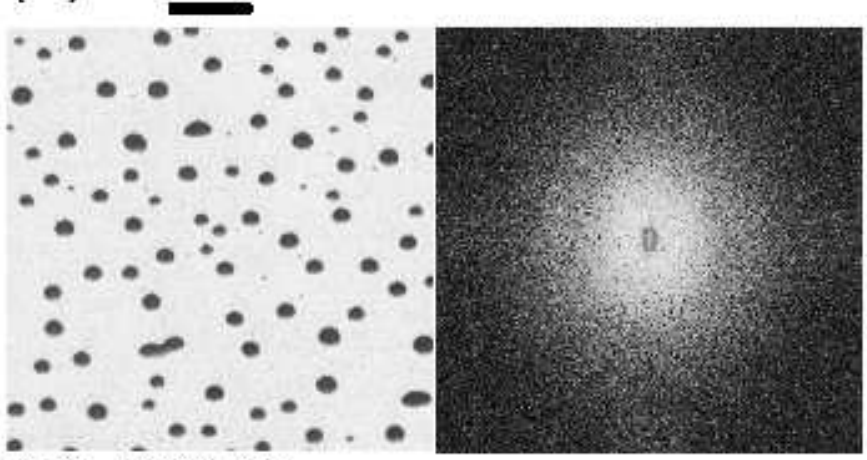

(D) $1000 \mathrm{~nm}$

Figure 1: 

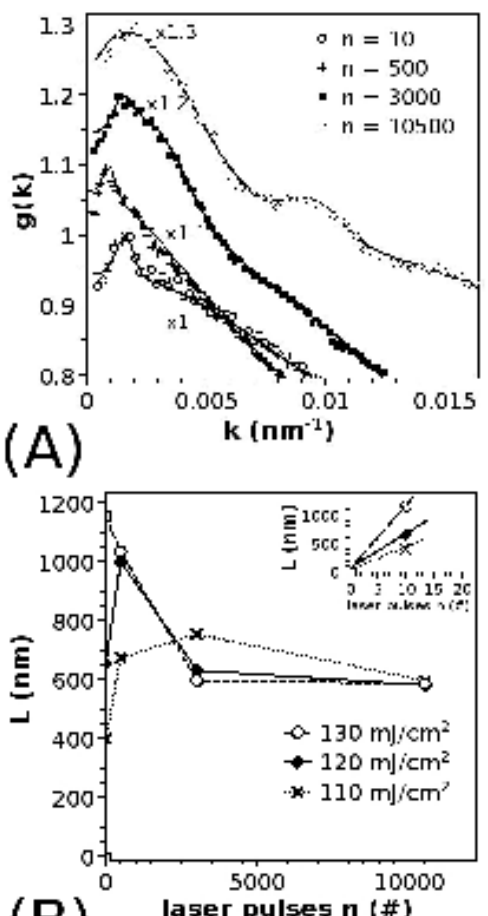

(B) laser pulses n (\#)
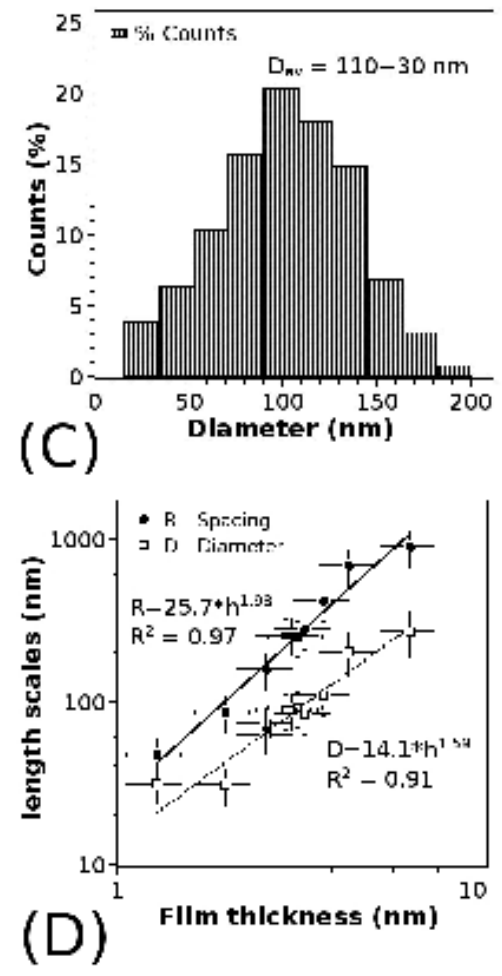

Figure 2: 